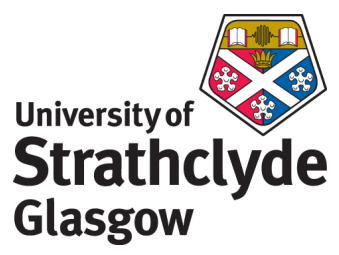

Al-Hanafy, Waleed and Weiss, S. (2009) Comparison of precoding methods for broadband MIMO systems. In: IEEE 3rd International Workshop on Computational Advances in Multi-Sensor Adaptive Processing, CAMPSAP 09, 13-16 December 2009, Aruba, Dutch Antilles.

http://strathprints.strath.ac.uk/27154/

Strathprints is designed to allow users to access the research output of the University of Strathclyde. Copyright $(C$ and Moral Rights for the papers on this site are retained by the individual authors and/or other copyright owners. You may not engage in further distribution of the material for any profitmaking activities or any commercial gain. You may freely distribute both the url (http://strathprints.strath.ac.uk) and the content of this paper for research or study, educational, or not-for-profit purposes without prior permission or charge. You may freely distribute the url (http://strathprints.strath.ac.uk) of the Strathprints website.

Any correspondence concerning this service should be sent to The Strathprints Administrator: eprints@cis.strath.ac.uk 


\title{
Comparison of Precoding Methods for Broadband MIMO Systems
}

\author{
Waleed Al-Hanafy ${ }^{\star \ddagger}$ and Stephan Weiss ${ }^{\star}$ \\ * Centre for Excellence in Signal \& Image Processing, Dept. of EEE, Univ. of Strathclyde, Glasgow, Scotland, UK \\ $\ddagger$ Electronics \& Communications Engineering Dept., Faculty of Electronic Engineering, Menoufia Univ., Menouf, Egypt \\ Email: \{waleed.alhanafy, stephan.weiss\} @eee.strath.ac.uk
}

\begin{abstract}
In this paper we investigate non-linear precoding solutions for the problem of broadband multiple-input multipleoutput (MIMO) systems. Based on a broadband singular value decomposition (BSVD) we can decouple a broadband MIMO channel into independent dispersive spectrally majorised singleinput single-output (SISO) subchannels. Bit loading is proposed to optimally utilise these SISO subchannels after mitigating their individual inter-symbol-interference (ISI) using TomlinsonHarashima precoding (THP). This method is benchmarked against recent results of both MMSE linear and THP designed for frequency-selective MIMO channels. Simulation results show that better bit-error-ratio (BER) can be achieved especially for higher throughput targets when compared to the benchmark.
\end{abstract}

\section{INTRODUCTION}

Modern applications of wireless communications often feature by high-rate transmission requirements. Therefore systems with high spectral efficiency characterisation incorporating multiple antennas at both transmit and receive side of the communication link, referred to as multiple-input multiple-output (MIMO) have recently received considerable attention [1], [2]. The communication channel of these systems cannot be considered as frequency-flat channel and hence incurs intersymbol-interference (ISI) along with co-channel-interference (CCI). Typical non-block based precoding/equalisation approaches including mostly decision feedback equalisation [3], V-BLAST approaches [4] adopted for the broadband case or a mixture thereof [5], as well as Tomlinson-Harashima precoding (THP) [6], [7]. These methods do not require a guard interval, can be globally optimised w.r.t. to e.g. mean squared error, and can therefore potentially achieve a higher spectral efficiency. The drawback of these schemes is the large effort in determining the optimum detection order in both space and time, often motivating the adoption of suboptimal approaches [4], [7].

Therefore, we propose a non-block based approach, which is based on a generalisation of the SVD - optimal in many ways to enable communication over a narrowband MIMO channel [8] — to the broadband case. A recently proposed broadband SVD (BSVD) [9] is applied to decouple the broadband MIMO system into frequency-selective single-input single-output (SISO) subchannels of ordered quality, similar to [10]. These broadband SISO subchannels are individually equalised using nonlinear THP with a variable transmission rate that best matches the individual qualities of respective subchannels, whereby the decision delay can be independently optimised for every subchannel.

The approach investigated in this paper is based on preliminary work in [10], which we extend by a more balanced implementation of THP between feedforward and feedback components, achieving an improved system performance. We also exploit spectral majorisation by a heuristic bit loading approach to demonstrate that an improved BER performance can be achieved when compared to a state-of-the-art broadband MIMO technique in [7] under the constraints of identical data throughput and transmit power.

\section{System Setup and Benchmark Description}

A broadband transceiver system between $N$ transmit and $M$ receive antennas is assumed in this study. This type of system is often described by a MIMO frequency selective channel with finite impulse response (FIR) that incurring both CCI in the space dimension as well as ISI in the time coordinate such that

$$
\mathbf{H}(z)=\sum_{l=0}^{L} z^{-l} \mathbf{H}_{l},
$$

where $L$ is the FIR length and $\mathbf{H}_{l}$ is an $M \times N$ matrix representing the channel at the $l^{\text {th }}$ time slice with coefficients $h_{m n}(l)$ being the complex baseband channel coefficient from the $n^{\text {th }}$ transmit antenna to the $m^{\text {th }}$ receive antenna at time index $l$.

Decision feedback equalisation (DFE) in SISO systems cancels ISI caused by already detected symbols by subtracting out their contribution from the received signal. DFEs have been applied to MIMO systems, where in addition to interference from already detected past data symbols, interference from spatially already detected symbols can be eliminated. In this case the optimum detection order - in time and space - is important if a good performance is to be attained. The authors in [7] jointly optimised ordering in both space and time and resulting in a MIMO THP precoder designed with MMSE criterion which we will use as a benchmark to our proposed method.

In this work we follow a different scenario, using the BSVD algorithm this problem can be reduced into some independent dispersive spectrally majorised SISO subchannels. We thereafter apply a transmission scheme with bit loading to 
optimally utilise these SISO subchannels after mitigating their individual ISI using THP [11], [12].

\section{Proposed Method}

In this section, mitigation of CCI using the BSVD algorithm resulting in a number of individual SISO subchannels is first presented. ISI caused by these SISO subchannels is then considered using the THP algorithm in conjunction with distributing transmission rates according to the individual qualities of the SISO subchannels as outlined in Fig. 2.

\section{A. Broadband SVD Review}

The application of a broadband singular value decomposition algorithm detailed in [9] to the channel matrix $\mathbf{H}(z)$ in (1) leads to a decomposition

$$
\mathbf{H}(z)=\mathbf{U}(z) \boldsymbol{\Sigma}(z) \tilde{\mathbf{V}}(z)
$$

with paraunitary matrices $\mathbf{U}(z)$ and $\tilde{\mathbf{V}}(z)$ and an approximately diagonalised and spectrally majorised matrix $\boldsymbol{\Sigma}(z)$. This decomposition is achieved by an iterative algorithm, which in each step eliminates the largest off-diagonal element by a delay step and a Givens rotation [9]. The algorithm has been shown to converge by transferring the energy of the channel matrix onto the main diagonal, and the approximation is due to limiting the number of algorithmic steps and the order of the resulting polynomial matrices $\mathbf{U}(z), \mathbf{V}(z)$ and $\boldsymbol{\Sigma}(z)$. The iteration steps are defined such that both $\mathbf{U}(z) \in \mathbb{C}^{M \times M}(z)$ and $\mathbf{V}(z) \in \mathbb{C}^{N \times N}(z)$ are paraunitary (or lossless) by definition, i.e.

$$
\begin{aligned}
\mathbf{U}(z) \tilde{\mathbf{U}}(z) & =\mathbf{U}(z) \mathbf{U}^{H}\left(z^{-1}\right)=\mathbf{I} \\
\tilde{\mathbf{U}}(z) \mathbf{U}(z) & =\mathbf{I} .
\end{aligned}
$$

The matrix $\boldsymbol{\Sigma}(z) \in \mathbb{C}^{M \times N}(z)$ in the limit diagonal

$$
\boldsymbol{\Sigma}(z)=\operatorname{diag}\left\{\boldsymbol{\Sigma}_{0}(z), \boldsymbol{\Sigma}_{1}(z), \cdots \boldsymbol{\Sigma}_{\min (M, N)-1}(z)\right\} .
$$

The diagonalisation can be ambiguous and has to be tied down by an additional constraint. As an extension of the ordering of singular values in a standard SVD, the algorithm in [9] aims to spectrally majorise $\boldsymbol{\Sigma}(z)$, such that

$$
\boldsymbol{\Sigma}_{0}\left(e^{j \Omega}\right) \geq \boldsymbol{\Sigma}_{1}\left(e^{j \Omega}\right) \geq \cdots \geq \boldsymbol{\Sigma}_{\min (M, N)-1}\left(e^{j \Omega}\right) \forall \Omega .
$$

Note that due to the iterative nature and the finite number of steps of the algorithm in [9], (5) and (6) may only be approximately fulfilled.

\section{B. Mitigation of Co-Channel Interference}

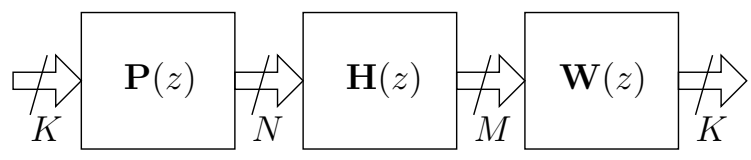

Figure 1. Co-Channel interference mitigation with precoder $\mathbf{P}(z)$ and equaliser $\mathbf{W}(z)$.
If channel state information is available at both receiver and transmitter, then the paraunitary matrices $\mathbf{U}(z) \in \mathbb{C}^{M \times M}$ and $\mathbf{V}(z) \in \mathbb{C}^{N \times N}$ can be utilised to obtain a linear equaliser and precoder as depicted in Fig. 1. If

$$
\begin{aligned}
\mathbf{U}(z) & =\left[\begin{array}{llll}
\underline{U}_{0}(z) & \underline{U}_{1}(z) & \cdots & \underline{U}_{M-1}(z)
\end{array}\right] \\
\mathbf{V}(z) & =\left[\begin{array}{llll}
\underline{V}_{0}(z) & \underline{V}_{1}(z) & \cdots & \underline{V}_{N-1}(z)
\end{array}\right]
\end{aligned}
$$

then with $K=\min (M, N)$ the precoder and equaliser are defined as

$$
\begin{aligned}
& \mathbf{P}(z)=\left[\begin{array}{llll}
\underline{V}_{0}(z) & \underline{V}_{1}(z) & \cdots & \underline{V}_{K-1}(z)
\end{array}\right] \\
& \mathbf{W}(z)=\left[\begin{array}{c}
\tilde{U}_{0}(z) \\
\underline{\tilde{U}}_{1}(z) \\
\vdots \\
\underline{\tilde{U}}_{K-1}(z)
\end{array}\right]
\end{aligned}
$$

to obtain

$$
\begin{aligned}
& \tilde{\mathbf{V}}(z) \mathbf{P}(z)= \begin{cases}\mathbf{I}_{N} & M \geq N \\
{\left[\begin{array}{c}
\mathbf{I}_{M} \\
\mathbf{0}_{(N-M) \times M}
\end{array}\right]} & M<N\end{cases}
\end{aligned}
$$

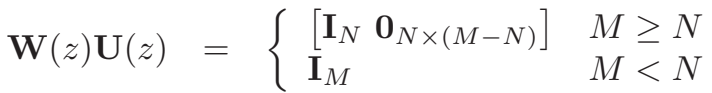

such that

$$
\underline{Y}(z)=\boldsymbol{\Sigma}(z) \underline{X}(z)
$$

With $\boldsymbol{\Sigma}(z)$ being approximately diagonal, the MIMO system has been decoupled and co-channel interference is suppressed.

\section{Individual ISI Mitigation}

Once the co-channel interference is mitigated we end up with a diagonalised system with spectrally majorised individual dispersive SISO subchannels (13) as depicted in Fig. 2(a). The next task is to mitigate the effect of dispersion incurred by these SISO subchannels as well as to exploit the spectral majorisation of BSVD algorithm. To this end, a rate-scaled THP system is proposed whereby individual data layers are fashioned to achieve throughput that best matches their respective SISO subchannels by selecting from different squareQAM constellations (QPSK, 16-QAM, 64-QAM and 256QAM) while the weakest subchannel may or may not be used according to a transmission target.

Fig. 2(b) shows the THP system designed to mitigate ISI of the $i^{\text {th }}$ subchannel of Fig. 2(a). Given the $i^{\text {th }}$ subchannel $\boldsymbol{\Sigma}_{i}(z)$ which is usually non-minimum phase, the role of the feedforward filter $\mathbf{f}_{i}(z)$ with length $L_{\mathbf{f}}^{(i)}$ is to drive the end-toend discrete-time response $\boldsymbol{\Sigma}_{i}(z) \mathbf{f}_{i}(z)$ to a monic minimum phase system, while the task of the $L_{\mathbf{b}}^{(i)}$-tabs feedback filter $\mathbf{b}_{i}(z)$ is to completely remove the remaining postcursor of $\boldsymbol{\Sigma}_{i}(z) \mathbf{f}_{i}(z)$ by the iterative feedback loop of Fig. 2(b). These two filters along with the decision delay are computed for each $\boldsymbol{\Sigma}_{i}(z)$ using spectral factorisation theory, details can be found in [3], [13].

A suitable bit loading is assigned to each SISO subchannel according to its strength. With the spectral majorisation property (6) of the iterative BSVD algorithm, the resulting SISO 


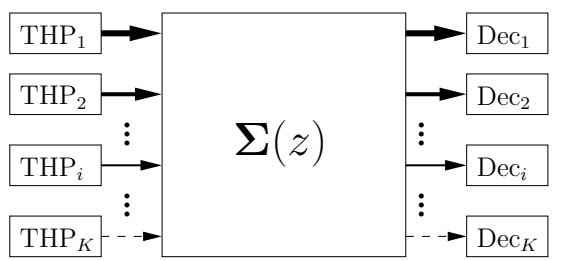

(a)

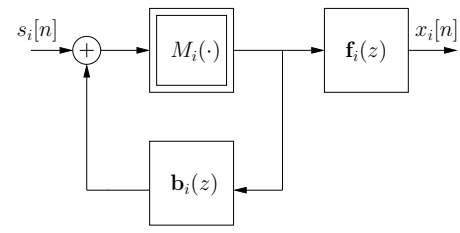

(b)

Figure 2. Equivalent model to Fig. 1 with THP applied for resulting SISO subchannels in (a), where $\operatorname{Dec}_{i}$ comprising both a modulo operator followed by a decision device. Details of $\mathrm{THP}_{i}$ block in (a) is given in (b).

subchannels demonstrated in the main diagonal of the decoupled system in (5) are ordered such that the first subchannel being the strongest one followed by the second subchannel and so forth [10]. It is also noted that the last subchannel is always unreliable and it is recommended to turn it off if a good performance is to be attained unless it is needed to achieve a target throughput, see Fig. 2(a). We choose to operate the proposed equivalent SISO system to achieve the same target throughput as its respective original MIMO system with details of the $i^{\text {th }}$ SISO-THP block in Fig. 2(a) given in Fig. 2(b).

\section{Simulation Results}

Computer simulations are conducted for a $4 \times 4$ MIMO system with a broadband channel of order $L=5$ and a power delay profile given in Table I where the channel coefficients $h_{m n}(l)$ are drawn from a complex Gaussian distribution with zero-mean and prescribed variance according to Table I. We will denote the benchmark system [7] described in Sec. II for both linear and THP precoding as MIMO-Lin and MIMOTHP, respectively. The length of the feedforward filter tabs for both MIMO-Lin and MIMO-THP systems as well as BSVDSISO system is set to twice the channel order i.e., 10 tabs for both MIMO systems and $L_{\mathrm{f}}^{(i)}=2 \times \operatorname{order}\left(\boldsymbol{\Sigma}_{i}(z)\right)$ for the $i^{\text {th }}$ BSVD-SISO subsystem, while the feedback filter length of each BSVD-SISO subsystem is set to the same order as its respective subchannel, i.e., $L_{\mathbf{b}}^{(i)}=\operatorname{order}\left(\boldsymbol{\Sigma}_{i}(z)\right)$. A comparison between our proposed model and the benchmark systems is set with the aim of achieving same system throughput. Three target throughput cases are considered, namely "8-bits", "16bits" and "24-bits". In each case, the overall throughput is achieved with our proposed BSVD-SISO model by distributing rates among individual SISO subchannels according to their strength while in the MIMO case is equally allocated by definition. We choose to assign these rates with two constraints: 1) discrete square-QAM constellations is only permitted and 2) transmission over the worst subchannel (last one) is avoided unless is needed to complete the target throughput. With these constraints a reasonable choice is made as shown in Table II.
For the first two cases, same throughput can be attained with the utilisation of the only first three subchannels while the fourth subchannel is switched off. However, for the "24bits" case the fourth subchannel has to be used to complete achieving the target throughput.

Table I

Channel Power Delay Profile

\begin{tabular}{|c|c|c|c|c|c|c|}
\hline path delay $($ symbol) & 1 & 2 & 3 & 4 & 5 & 6 \\
\hline relative power $(\mathrm{dB})$ & 0 & -4 & -8 & -12 & -16 & -20 \\
\hline
\end{tabular}

Table II

SISO-BSVD FOr SAmE Throughput TARget AS A 4X4 MIMO System

\begin{tabular}{|c|c|c|c|}
\hline Throughput & Caee1: 8-bits & Case2: 16-bits & Case3: 24-bits \\
\hline \hline MIMO & QPSK & 16-QAM & 64-QAM \\
\hline SISO-1 & 16-QAM & 64-QAM & 256-QAM \\
SISO-2 & QPSK & 64-QAM & 256-QAM \\
SISO-3 & QPSK & 16-QAM & 64-QAM \\
\hline SISO-4 & QPSK & QPSK & QPSK \\
\hline
\end{tabular}

Figures: 3, 4 and 5 demonstrate the BER performance (averaged over 300 different channel realisations) comparison between our BSVD-SISO and both MIMO-Lin and MIMOTHP for the three cases, respectively. The BSVD-SISO curve that representing same achieved throughput as the benchmark MIMO systems is marked with a solid-line in all of these figures. In Fig. 3 it is clear that average BER for the first two subchannels (marked as " $1-2$ ") is much better than both MIMO linear and THP with 2 bits $(25 \%)$ degradation in the overall throughput. For same achieved throughput comparison the average BER of the first three subchannels (marked as "1:3") is slightly higher than MIMO-THP $(<0.5 \mathrm{~dB}$ loss at $10^{-3}$ BER) and outperforms for higher SNR (or BER $<10^{-4}$ performance). If the fourth channel is switched on the overall throughput can be increased by a factor of $25 \%$ at the expense of very poor BER that dominates the overall performance (marked as "all"). Note that for the BSVD-SISO system, the BER of the combined first $k$ subchannels $\bar{P}_{k}$ is averaged over these individual subchannels BERs $P_{i}$ weighted by their respective allocated number of bits $b_{i}$, i.e., $\bar{P}_{k}=\frac{\sum_{i=1}^{k} b_{i} P_{i}}{\sum_{i=1}^{k} b_{i}}$

For higher throughput-achieving operation, "16-bits" and "24-bits" results are shown in Fig. 4 and Fig. 5, respectively. In Fig. 4, the BSVD-SISO system is clearly outperforms its counterpart MIMO benchmark for normal practical operating region (BER < 0.01). It is also noted very close BER for both averaged first two and first three subchannels which reveals the good choice of individual rates (modulations) that best match SISO subchannels strengths. Extra throughput can be achieved $(12.5 \%)$ if the fourth subchannel is turned on at the cost of almost $1.5 \mathrm{~dB}$ increase in medium SNR operation. Fig. 5 shows the performance comparison for the "24-bits" case, in this case all SISO subchannels has to be taken into account for same target throughput as the MIMO benchmark (see Table II). A gain of more than $2.5 \mathrm{~dB}$ in SNR can be achieved at BER $\leq$ 0.05 , also it is obviously noted the close performance of each combined BSVD-SISO subsystem which again demonstrates 


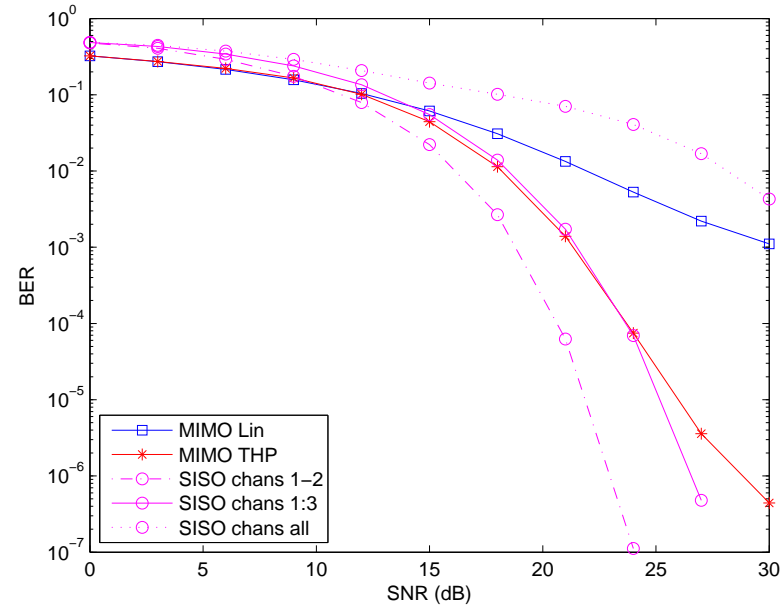

Figure 3. Case "8-bits" where the 1st SISO subchannel operates on 16-QAM and all other subchannels on QPSK modulation.

the good choice of individual rates allocated to each BSVDSISO subsystem.

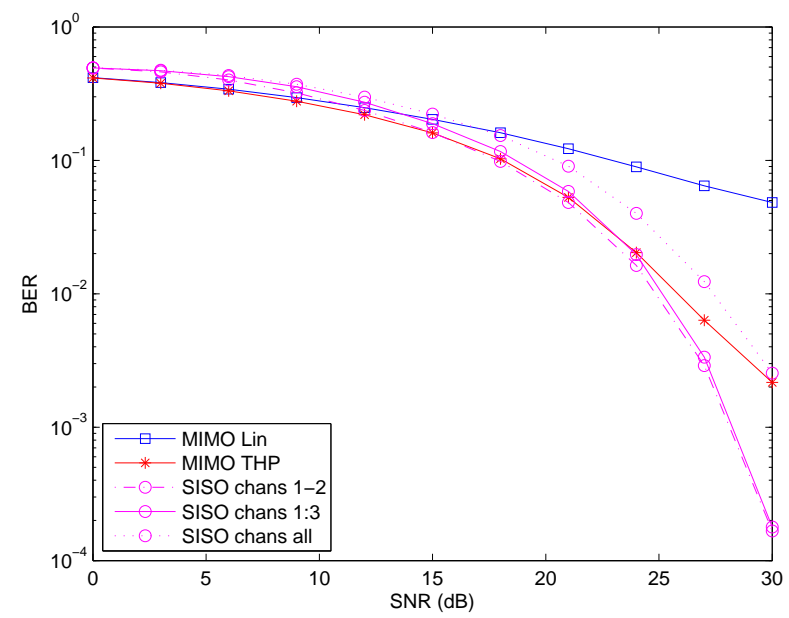

Figure 4. Case "16-bits" where the first two SISO subchannels operates on 64-QAM, 3rd one on 16-QAM and last subchannel on QPSK modulation.

\section{Conclusions and Future Research}

A broadband SVD (BSVD) based solution with variable transmission rates is proposed for the MIMO broadband channel precoding problem. This solution is achieved by two steps: firstly, the BSVD is applied to the MIMO frequencyselective channel resulting in a number of independent SISO subchannels with ordered qualities. Secondly, non-linear THP precoding algorithm with variable rates using predefined square-QAM constellations is applied to combat ISI incurred by these subchannels as well as achieving a target throughput constrained by an acceptable QoS and transmit power. Next

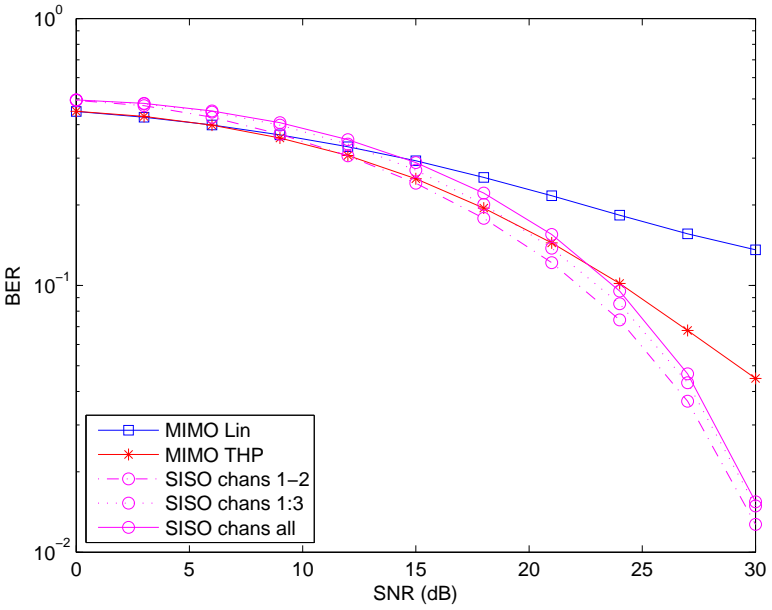

Figure 5. Case "24-bits" where the first two SISO subchannels operates on 256-QAM, 3rd one on 64-QAM and last subchannel on QPSK modulation.

research is expected to adaptively allocate these rates while keeping guaranteed QoS thresholds.

\section{REFERENCES}

[1] G. Foschini and M. Gans, "On Limits of Wireless Communications in a Fading Environment when Using Multiple Antennas," Wireless Personal Communications, vol. 6, no. 3, pp. 311-335, March 1998.

[2] I. E. Telatar, "Capacity of Multi-antenna Gaussian Channels," European Transactions on Telecommunications, vol. 10, no. 6, pp. 585-595, December 1999

[3] R. F. Fischer and J. B. Huber, "Equalization Strategies for Transmission over Space and Time," Electronik und Informationstechnik, vol. 6, pp. $187-195,2005$

[4] A. Lozano and C. Papadias, "Layered Space-Time Receivers for Frequency-Selective Wireless Channels," IEEE Transactions on Communications, vol. 50, no. 1, pp. 65-73, January 2002.

[5] L.-U. Choi and R. D. Murch, "A Pre-BLAST-DFE Technique for the Downlink of Frequency-Selective Fading MIMO Channels," IEEE Transactions on Communications, vol. 52, no. 5, pp. 737-743, May 2004.

[6] R. F. H. Fischer, C. Windpassinger, A. Lampe, and J. B. Huber, "Tomlinson-Harashima Precoding in Space-Time Transmission for LowRate Backward Channel," in International Zurich Seminar on Broadband Communications, 2002, pp. 7.1-7.6.

[7] M. Joham, D. A. Schmidt, J. Brehmer, and W. Utschick, "Finite-Length MMSE Tomlinson-Harashima Precoding for Frequency Selective Vector Channels," IEEE Transactions on Signal Processing, vol. 55, no. 6, pp. 3073-3088, June 2007.

[8] M. Vu and A. Paulraj, "MIMO Wireless Linear Precoding," IEEE Signal Processing Magazine, vol. 24, no. 5, pp. 86-105, September 2007.

[9] J. G. McWhirter, P. D. Baxter, T. Cooper, S. Redif, and J. Foster, "An EVD Algorithm for Para-Hermitian Polynomial Matrices," IEEE Transactions on Signal Processing, vol. 55, no. 5, pp. 2158-2169, May 2007.

[10] W. Al-Hanafy, A. P. Millar, C. H. Ta, and S. Weiss, "Broadband SVD and non-linear precoding applied to broadband MIMO channels," in 42nd Asilomar Conference on Signals, Systems and Computers, Oct. 2008, pp. 2053-2057.

[11] M. Tomlinson, "New Automatic Equaliser Employing Modulo Arithmetic," IEE Electronics Letters, vol. 7, no. 5/6, pp. 138-139, March 1971.

[12] H. Harashima and H. Miyakawa, "Matched-Transmission Technique For Channels with Intersymbol Interference," IEEE Transactions on Communications, vol. 20, pp. 774-780, August 1972

[13] R. F. H. Fischer, "Sorted Spectral Factorization of Matrix Polynomials in MIMO Communications," IEEE Transactions on Communications, vol. 53, no. 6, pp. 945-951, June 2005. 